\title{
A Multimedia Document Distribution System Over DQDB MANs*
}

\author{
Luis Orozco-Barbosa ${ }^{a}$ and Michel Soto ${ }^{b}$
}

a Department of Electrical Engineering, University of Ottawa, 161 Louis Pasteur, Ottawa, Ont., K1N 6N5 Canada

b Institut Blaise Pascal, Laboratoire MASI, Université Pierre et Marie Curie 4 Place Jussieu, 75252 PARIS Cedex 05, France

In this paper, we present a system architecture for supporting Real-time Multimedia Document Distribution Applications. This architecture is based on an extended version of the ODA (Office Document Architecture) standard using as communications support the IEEE 802.6 MAN Standard: an integrated services local area network (ILAN). Multimedia document delivery is performed by dynamically allocating the network bandwidth. This allocation strategy is based on the bandwidth required by the various media composing the documents. A performance analysis of the proposed bandwidth allocation scheme is given.

Keyword Codes: C.2.2, C.2.4, C.2.5

Keywords: Network Protocols; Distributed Systems, Local Networks

\section{INTRODUCTION}

Multimedia communications may be simply defined as the exchange of highly-structured documents including text, graphics, voice, audio and video sequences. Three main requirements could be identified for multimedia communications systems [1] :

- Multimedia document standards : The spread of multimedia applications makes possible the interchange of multimedia documents. This can only be achieved by means of well accepted international standards.

- Multimedia workstations : The use and integration of new peripherals capable of supporting a wide variety of multimedia applications, such as video-conferencing (digital telephony and video).

- High speed networks : High speed communications networks will have to provide the means to support a wide variety of communications services : bandwidth on demand, synchronous and asynchronous services, guaranteed quality of service (low response times and low blocking probabilities).

In the near future, large storage capacities and transmission technologies able to provide sufficient data bandwidth transfer will be both necessary to handle multimedia documents. This will be needed particularly for handling multimedia documents comprising digital video sequences. Several research axes on technologies, such as optical storage, file handling

\footnotetext{
* This work was supported in part by the Telecommunications Research Institute of Ontario (TRIO) and the Natural Sciences and Engineering Research Council of Canada under grant number OGPIN 334.
} 
techniques and non-volatile cache memory as applied to multimedia applications are being carried out by research laboratories and universities [1].

Multimedia information implementations are built up by combining a wide variety devices, technologies and procedures. For example, a multimedia information system in a medical environment will require the use of very large capacity storage devices, e.g., optical disks, high speed computer network, special acquisition devices and highly reliable protocols [2]. This kind of systems, multimedia medical information systems, will support digital imaging applications requiring high resolution images and lossless compression techniques. Higher compression rates may be used in other environments, such as multimedia office information system. In this last case, the amount of information to be stored may be reduced substantially.

Multimedia information systems often rely on a distributed architecture where several servers are used for storing multimedia documents. It is now a reality that working environments are already heterogeneous because they are composed of different types of devices going from fully equipped workstations to more or less powerful personal computers with low storage capacities.

Another important point is the need of a structuring and interchanging multimedia documents standard. These standards should define the links and synchronization of static and time-based information as well as the interchange of multimedia information. One of the most promising standard is ODA, the Office Document Architecture [3]. Some other relevant standards such as HyTime [4] and MHEG [5] may also be considered. However, these two later standards are in a committee draft and in a working document stage.

In this paper, we present a system architecture for supporting Real-time Multimedia Distribution Application. By Real-time Multimedia Distribution Application, we mean a system capable of providing the support for storage and on-line retrieval of multimedia documents from a multimedia server. As multimedia documents will be much bigger in size than simple ASCII text files, the retrieval of these documents are much likely to be carried on-line. In this paper, we propose a system architecture for supporting the real-time delivery of multimedia documents. This architecture is based on the CCITT Office Document Architecture (ODA) Standard and it uses as transmission support an integrated services local area network (ILAN).

The paper is organized as follows. Section 2 reviews the general architecture of the ODA Standard. In Section 3, we provide a functional description of bandwidth allocation strategy. Section 4 contains a performance analysis of the proposed strategy aiming to assess the bandwidth required for supporting multiple multimedia connections. Finally, in Section 5 our conclusions and future research directions are given.

\section{MULTIMEDIA DOCUMENT ARCHITECTURE}

\subsection{Standards}

The definition of the multimedia document architecture is a fundamental element in a multimedia communications system. Current effort are being carried to define a standard able to specify the different types of data needed for use in multimedia systems [6][7]. It has been recognized that one of the best way to approach this problem will be to define a standard that could be easily extended to accomodate additional formats [6]. Another approach has been to extend current standards [8]. In our approach, we have preferred to look into current standards. We believe that current standards, such as ODA provide the modelling basis required for the definition of a multimedia documents architecture. Furthermore, it has been recognized that whatever standards will be adopted, multimedia communications systems having different characteristics will have to be able to interwork. We therefore look into the particular case of a multimedia document architecture based on an extended version of ODA [3].

ODA has been studied in the context of office applications. The types of documents that may be represented in the ODA document model are those frequently used in the general office work 
such as reports, letters, forms, invoices, memoranda. In practice such office documents may contain text, graphics and pictures within a single document: a so called "Multimedia Document". The purpose of the document model is to provide a method of describing the electronic representation of these documents, including the type of information found in their content. The structured description of the documents is termed the "architecture" of the document. A document architecture can be subdivided in three categories, namely, logical structure, layout structure and content .

The logical structure provides a method for organizing the content of the document and it is intended to closely correspond to those aspects of the document structure related to the functional semantic of the document. The layout structure provides a method for organizing the content of the document into pages and areas within pages. The details on the document architecture are given in [3].

Multimedia document may contain information such as text, graphics and images. This information may be organized as follows:

- a header which contains information related to the originator and recipient of the document such as name, document title and date .

- a control part containing the scenario, i.e., the description of the existing links between the various document's components.

- the body of the message. Each body part will contain one or various formats.

The goal of the document architecture is to propose means by which the document generation and reading scenarios can be represented and described with respect to the logical structure and the synchronization requirements between body parts.

\subsection{An ODA-based Multimedia Document Architecture}

The multimedia document architecture defines the method for generating and reading documents. The reading of the document by the recipient is performed in an animated fashion. The term animation is used to express the simultaneous treatment (e.g. display, playback) of the different body parts.

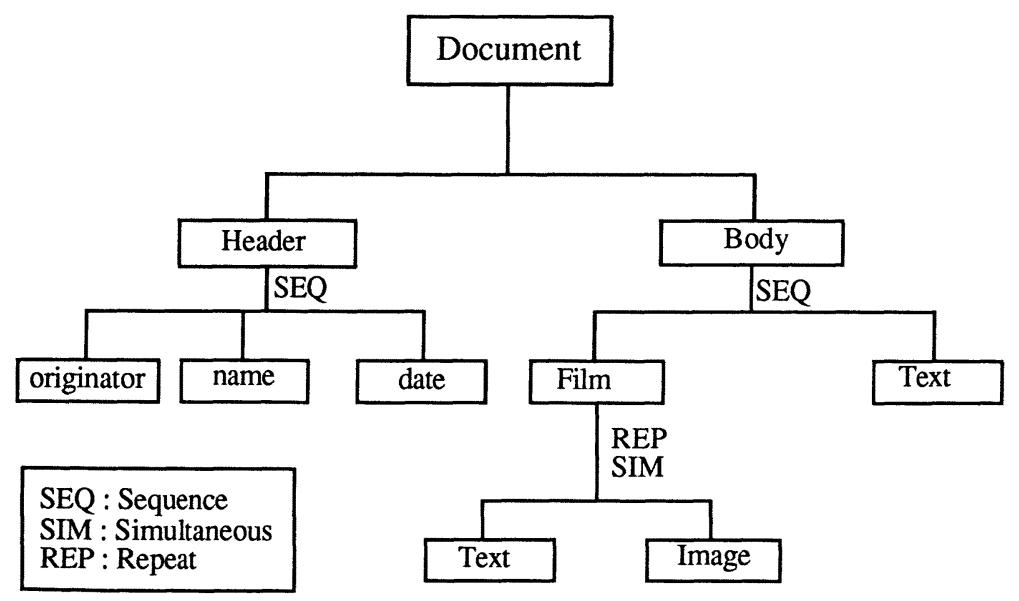

Fig. 1 Generic logical structure of a multimedia document 
The generic logical structure of the document specifies the set of rules by which the document is to be constructed. The generic structure is very similar to the concept of a class in object oriented programming languages and indeed the generic structure of a document is referred to as its class. Fig. 1 shows the generic structure for a typical multimedia document. This structure, however, is given as an example of possible document organizations.

In Fig. 1 the generic structure defines a document as a sequence containing a Header and a body. The header in turn is composed of a originator identifier, name, date of the document. This kind can be further extended to contain other relevant information. The body of the document is a set of parts. The figure does not show the control part. As already stated, this control part will contain the information regarding the links among the different body parts. In particular, it will include all the operators defining the relation among the various body parts.

The film section consists of a text and an associated image. The text may be related to the image as a whole. In this case the text is used for this purpose. If a more detailed association is necessary, a window section can be used, first to indicate the particular portion of the image to be referred (the position may be located, in the image, by means of a reference to the particular portion for example).

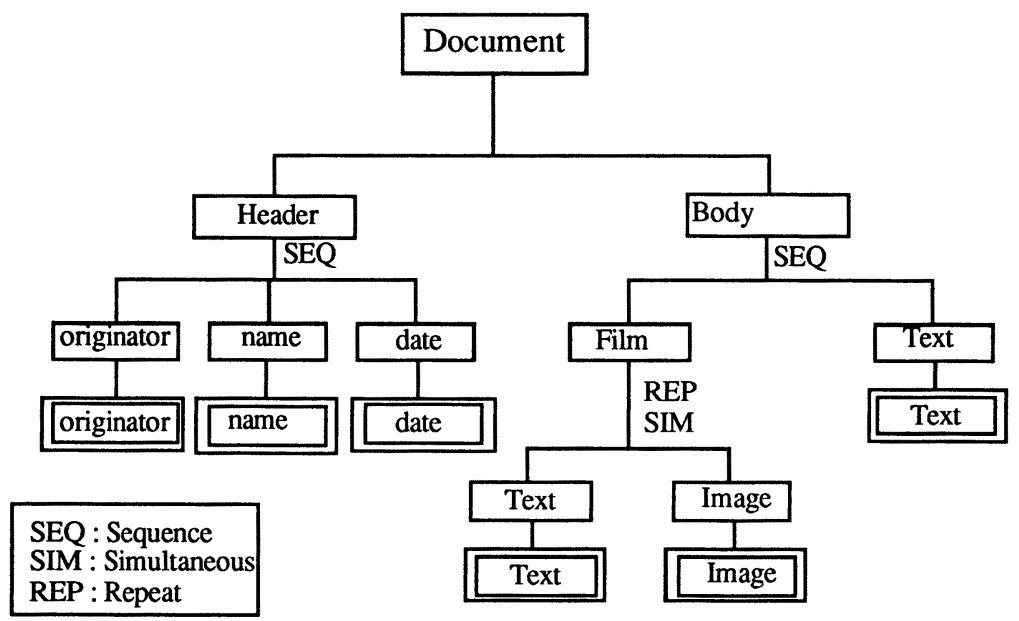

Fig. 2 Generic logical structure of a multimedia document

The specific structure of the document is derived from the generic structure. Fig. 2 represents an instance of a specific logical structure generated, with respect to the generic logical structure. Content portions are located at the lowest level components of the tree. Content architecture defined in the current version of ODA may be used for text, geometric and raster graphics. However for bulky data, such as video sequences further extensions to the current ODA standard are needed. Some experimental implementations have overcomed this limitation by placing bulky data out of the document architecture by replacing them by references [9]. 


\section{REAL-TIME MULTIMEDIA DOCUMENT DISTRIBUTION}

The various elements of a real-time multimedia system architecture have to interact in order to properly carry out the distribution of documents. In the following, we give a description of the functions to be carried by network bandwidth allocation scheme. In this description, we also explain the way the allocation scheme makes use of the information describing the organization of the multimedia document (scenario). This is a central element enabling the proper allocation of the bandwidth following the requirements of the application (transfer of multimedia documents). We also describe the way these elements interact with all the other service elements and the communication services provided by DQDB.

\subsection{Scenario}

As already mentioned, the scenario describes the organization of a multimedia document. This description provides a temporal as well as a spatial relation of the different elements of a multimedia document. Figure 3 depicts an example of a scenario of a multimedia document composed by three different media, namely text, voice and video. The scenario contains all the required information to playback the multimedia document. For the particular example depicted in Fig. 3, the scenario corresponds to a multimedia document which starts by a video segment of 3 minutes, followed by a segment of voice and text of 3 minutes, a pause of 2 seconds and ending with a voice and video segment of 5 minutes. From the example, we can distinguish the two main temporal relation depicted in the example of Figures 1 and 2: simultaneous and sequential. The scenario also provides us with the bandwidth requirements of the various multimedia segments. This information is particularly useful for playing back the multimedia document as the retrieval of the document from a multimedia database server takes place. The main challenge is to allocate the bandwidth to make possible the timely transfer of the different elements of the document. This is the purpose of defining a bandwidth allocation scheme using the information provided by the scenario.

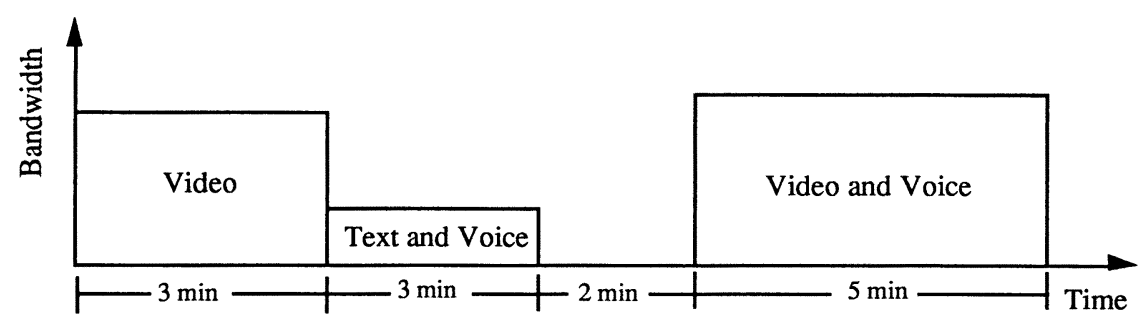

Fig. 3 A Typical Scenario

\subsection{Network Bandwidth Allocation Scheme}

In our architecture, the allocation of the network bandwidth is dynamically performed. One of the major challenges is therefore to define a Network Bandwidth Allocation Scheme (NBAS) suitable to handle in real-time the requests for allocating as well as freeing the network bandwidth. The operation of NBAS should be closely related to the services provided by the network. In this study, we have focused on the use of the IEEE 802.6 standard [10]. DQDB (Distributed Queue Dual Bus) has been selected by the IEEE 802.6 working group as the standard for Metropolitan Area Networks (MANs). The timing structure of DQDB is based on 
frames of $125 \mu \mathrm{s}$ comprising a given number of fixed slots. In turn, each DQDB slot comprises 48 (voice) channels with a nominal bit rate of $64 \mathrm{kbps}$ per channel. Slots are accessible by two methods :

- In the pre-arbitrated (PA) access, slots are marked by the node that is head of the bus. These slots carry isochronous service octets at defined offsets, every $125 \mu \mathrm{s}$.

- In the queues arbitrated (QA) access, slots are assigned by means of a queueing mechanism for outstanding slots. A queue is maintained in a distributed manner through all the active nodes within the MAN. Three levels of priority are supported by this method.

In order to adapt the two aforementioned access methods to the higher levels the following Convergence Functions (CF) have been included in the DQDB standard:

- Isochronous Convergence Function (ICF)

This function has specially been designed for connection-oriented services requiring timing relation between the source and the destination, such as constant bit rate.

- MAC Convergence Function (MCF)

This function provides the basis for connectionless communications. The destination and source addresses are included in the header of every MAC protocol data unit.

- Connection Oriented Convergence Function (COCF)

This function has not been completely defined in the standard. However provisions are contained for its future definition.

- Other Convergence Functions (OCF)

The inclusion of these functions will allow to define new services.

In order to provide the service required to dynamically allocate the bandwidth for retrieving, we define a new convergence function. The main reason behind our choice of defining a new convergence is twofold, 1) under this scheme, a multimedia document is progressively transmitted by means of multimedia segments; 2 ) the definition of a dynamic bandwidth scheme looks suitable for optimizing the bandwidth utilization. In the next section, we will demonstrate the advantages of using this scheme.

Fig. 4 shows the protocol architecture relating Network Bandwidth Allocation Entity (NBAE) to the new OCF. The main objective of the NBAE is to coordinate the operation of the application (not shown in the figure) and the convergence function. The functions to be performed by the NBAE are : to interpret the multimedia document scenario and upon this interpretation to drive the convergence function in allocating the bandwidth needed for conveying the document accordingly.

In the following, the operation of the proposed bandwidth allocation scheme will be explained by using the scenario depicted in Fig. 3. In order to convey all the information needed to indicate to the Head Of Bus (HOB) how to mark the slots, the NBAE will use, through the OCF, the PA slot with Virtual Channel Identifier (VCI) all ones defined in the DQDB to convey all the required signaling information [10]. We will refer to this slot as the Sslot and in order for the scheme to operate properly, the S-slot should precede the QA slots of the current cycle. The 48-bit payload of the S-slot is divided into three fields, namely F-0, F-1 and F-2. These fields are initially set up to all zeros by the HOB. As the S-frame passes by the nodes, the NBAE will request the OCF to reserve a given number of slots according to the rules depicted in Table 1 . The number of slots is reserved by incrementing the fields F- 0 , F-1 and F-2 which corresponds to the one of three levels of priority with priority 2 being the highest one. According to this scheme, NBAE requests with the lowest priority the allocation of corresponding number of slots indicated by F-0. The priority one is used at the beginning of a new segment. For instance, at the beginning of the multimedia segment consisting of text and voice, the NBAE will direct OCF to allocate the required number of slots with a priority one. Finally, the highest priority, number 2 , is used when a session is engaged in transferring a 
multimedia segment. Upon receiving this information and for a given cycle, the HOB will start by allocating the slots with priority 2 , followed by 1 and finally 0 .

The rationale to define three priorities can be simple explained as follows. As the number of slots per cycle is finite, the number of active sessions may exceed the number of slots available. Therefore, by assigning the highest priority to the session currently engage in the transfer of segment, we attempt to provide to the user with a synchronous transfer of the required information. The second level of priority will give an advantage to the session already in progress but initiating a new segment which may be slightly delayed without affecting the quality of service provided to the user. Finally, the third level of priority will make sure that new sessions will be initiated only if enough bandwidth to provide the quality of service required is available.

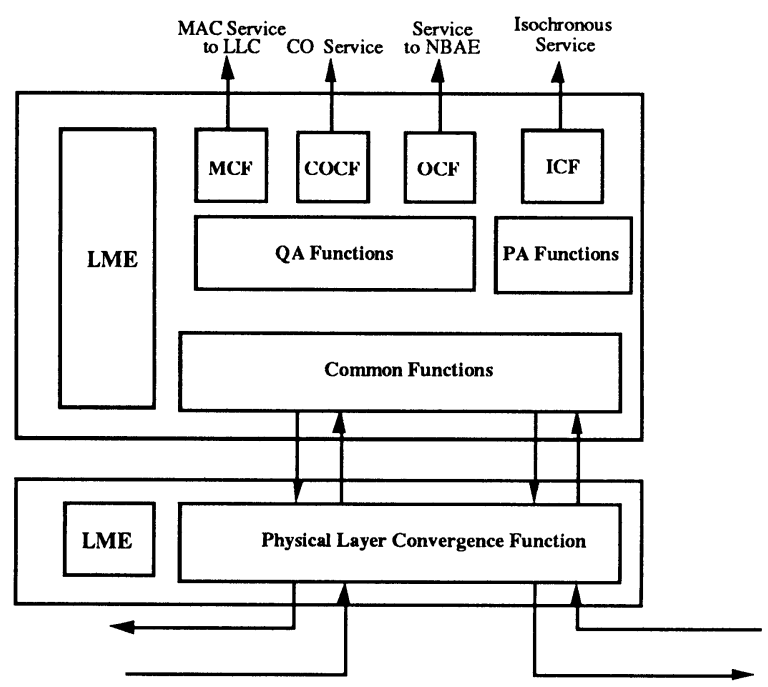

Fig. 4 DQDB Node Functional Architecture

Table 1 Bandwidth Allocation Priorities

\begin{tabular}{cl}
\hline Priority & Condition \\
\hline 0 & New Session \\
1 & Beginning of New Segment \\
2 & Segment in Progres \\
\hline
\end{tabular}




\section{PERFORMANCE STUDY}

The architecture suggested in this paper aims to optimize the use of the bandwidth of the underlying communication network by taking into consideration the quality of service required by the real-time multimedia application. It is particularly important to evaluate the amount of bandwidth and the blocking probability required for providing service to a large number of users. Blocking may result during the delivery of a document mainly by lack of bandwidth. From the user point of view, it will be very annoying to be blocked in the middle of a document delivery. It is therefore essential for the service provider to guarantee a low blocking probability.

\subsection{Analytical Model}

The bandwidth request process can be modeled by using a closed, multi-class queueing network (Fig. 5). The service rates, $\mu_{\text {NODE }_{i}}$ of the infinite servers $\operatorname{NODE}_{i}, i=1,2, \ldots N$, represent the interarrival rates of the connection requests. Servers SIGNAL and HOB describe the signalling channel and the Head Of the Bus, respectively. By signalling channel, we mean the PA slot used for conveying the requests for slots. The communication active period is modelled by infinite servers whose service rates, $\mu_{\text {TRAFFIC }}{ }_{i}$, are set according to the length (holding time) of the various HBS's. By using this model, it is possible to carry out an analysis of the bandwidth allocation scheme under various conditions. The model can be solved by applying the numerical method Convolution[11].

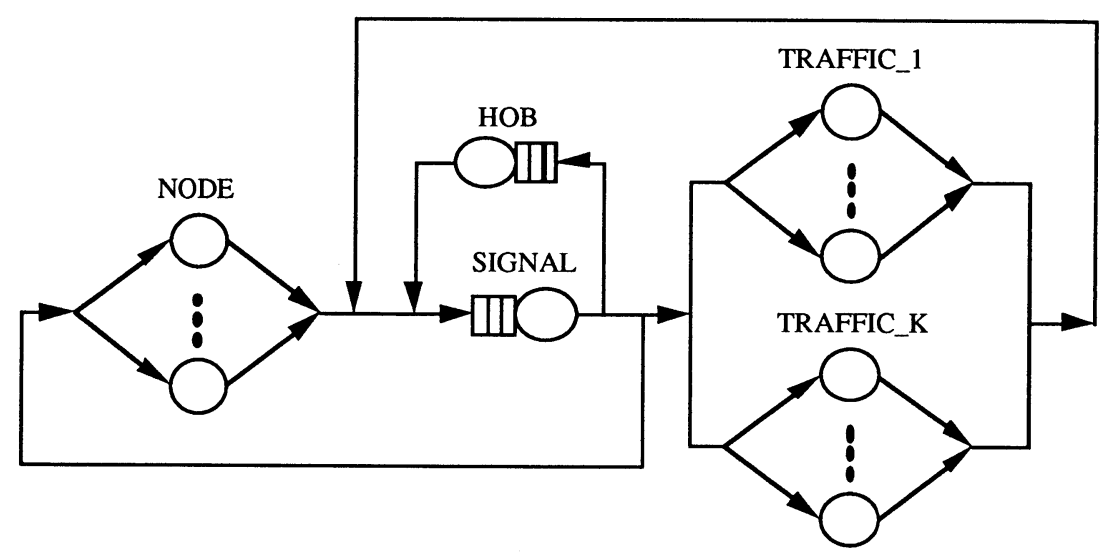

Fig. 5 Queueing Model of the Bandwidth Allocation Scheme

The number of channels used for the transmission of a multimedia document will vary from a segment to another. The blocking probability, $\mathrm{p}_{\mathrm{B}}$, can be expressed by :

$\mathrm{p}_{\mathrm{B}}=\mathrm{p}\left(\mathrm{y}_{\mathrm{i}}>\mathrm{K}\right)$ 
where $y_{i}$ is the number of segment in service and is given by :

$\mathrm{y}_{\mathrm{i}}=\left(\mathrm{n}_{\mathrm{i} 1}, \mathrm{n}_{\mathrm{i} 2}, \ldots \mathrm{n}_{\mathrm{iR}}\right)$

where $\mathrm{R}$ and $\mathrm{n}_{\mathrm{ir}}$ are the total number of different classes of segment and the number of segment of class $r$ in service center $i=$ TRAFFIC, respectively. The parameter $\mathrm{K}$ is given by :

$$
\mathrm{K}=\left\lceil\mathrm{Q} / \sum_{\mathrm{j}=1}^{\mathrm{R}} \mathrm{s}_{\mathrm{ij}}\right\rceil
$$

where $s_{i j}$ and $Q$ are the number of channels used per type of HBS at server $i=$ TRAFFIC, and the total number of channels in the system, respectively. $\lceil\mathrm{T}\rceil$ is defined as the smallest integer not less than the real number $\mathrm{T}$.

The blocking probability due to lack of bandwidth can be calculated by first determining the equilibrium state probabilities at server TRAFFIC. These probabilities, according to the queueing theory [11], are given by :

$$
P\left(S=\left(y_{1}, y_{2}, \ldots y_{N}\right)\right)=G g_{1}\left(y_{1}\right) g_{2}\left(y_{2}\right) \ldots g_{N}\left(y_{N}\right)
$$

where $S$ is an aggregate state of the system and $G$ is a normalizing constant chosen to make the equilibrium state probability sum to 1 , respectively. For the case of a infinite server (IS) service center :

$$
\mathrm{g}_{\mathrm{i}}\left(\mathrm{y}_{\mathrm{i}}\right)=\prod_{\mathrm{r}=1}^{\mathrm{R}}\left(1 / \mathrm{n}_{\mathrm{ir}} !\right)\left[\mathrm{e}_{\mathrm{ir}} / \mu_{\mathrm{ir}}\right]^{\mathrm{n}_{\mathrm{ir}}}
$$

where $e_{i r}$ and $\mu_{i r}$ are the relative arrival rate of class $r$ customers to service center $i$ and the mean service rate of class $r$ customers at service center $i$, respectively.

The blocking probability is therefore given by :

$P_{B}=\sum_{S(N, i)} P\left(S=\left(y_{1}, y_{2}, \ldots y_{N}\right)\right)$

where the state space, $\mathrm{S}(\mathrm{N}, \mathrm{i})$, for $\mathrm{N}$ customers at the ith queue is given by :

$S(N, i)=\left\{y_{i}=\left(n_{i 1}, n_{i 2}, \ldots n_{i R}\right) \mid \sum_{j=1}^{R} s_{i j} n_{i j}>Q\right\}$ 


\subsection{Numerical Results}

In this section, we illustrate the performance of the proposed bandwidth allocation scheme. Our main aim is to show the required bandwidth for supporting multiple multimedia document deliveries, as well to evaluate the blocking probability which may result during the document delivery.

In order to illustrate the performance, we have assumed that the documents consist of two media, namely, audio and video. Audio sources require a sustained bit rate of $772 \mathrm{kbps}$, that is to say 12-bytes at every DQDB frame [11], while video sources require a bit rate of $1.544 \mathrm{Mbps}$. This latter value is in compliance with the current CCITT's Recommendation H.261 (also called pX64) for visual telephony (videophone and vide-conferencing) [12]. It is assumed that the multimedia documents are transmitted by using two different classes of segment, namely SEG_mm and SEG_audio. The former corresponds to an HBS comprising both media, while the latter only consists of audio. We assume a nominal bit rate of $44.736 \mathrm{Mbps}$ for DQDB, corresponding to $14 \mathrm{DQDB}$ slots (672 voice channels). This value has been suggested as a prime candidate for a network speed compatible with DS3 circuits [10]. Assuming the aforementioned values and a static bandwidth allocation scheme is easy to verify that the maximum number of concurrent multimedia document deliveries is limited to 18.

By now, it is difficult to predict the ratio of the various media composing a multimedia document. It is however expected that the development of new technology will make common practice the use of visual (video and images) services [1][13][14]. In order to better evaluate the performance of the proposed bandwidth allocation scheme, we have divided our study into two parts. First, the use of video has been limited to half of the multimedia document contents, while in the second part the extensive use of video has been assumed. Furthermore, it is worth to notice that typically the service times for the different segments are several orders of magnitude higher than the signalling and processing to be carried out by the head-end node.

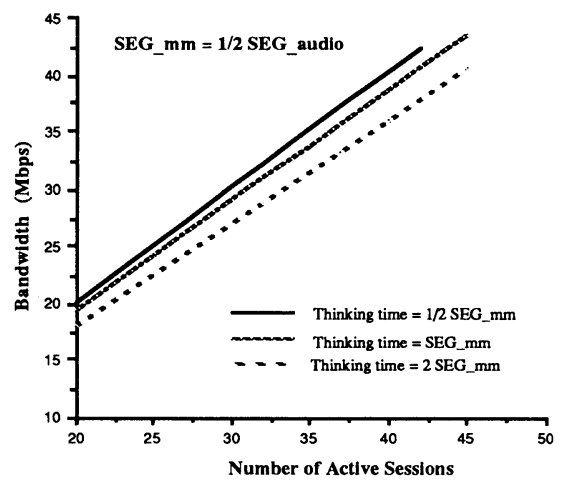

Fig. 6 Bandwidth vs. Number of Active Sessions

Fig. 6 shows the bandwidth required for supporting the concurrent retrieval of multimedia documents. In this case, the extensive use of audio service is assumed, while the use of video only accounts for a third of the total document length. This bandwidth has been evaluated for three different thinking times (time between two successive requests). These values range from half to twice of the service (holding) time of the SEG_mm. For all three cases, the figure shows an important improvement in the number of concurrent active retrieval sessions as opposed to the static bandwidth allocation scheme of 18 . For all three cases, the improvement is in the order of $150 \%$. 


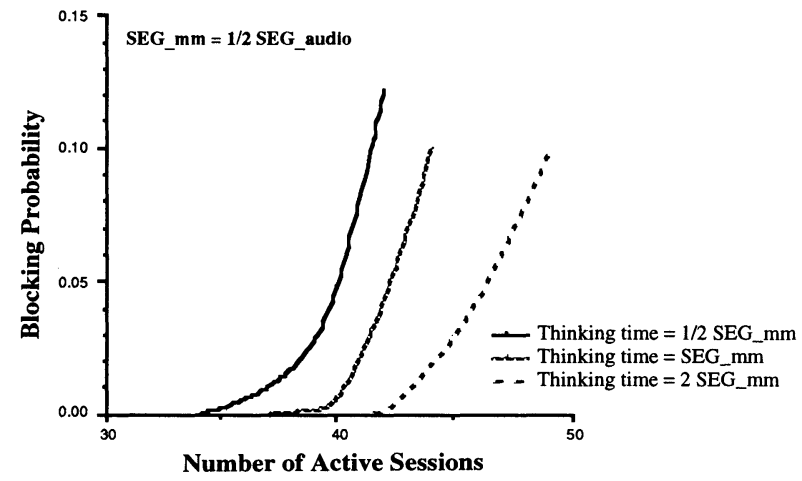

Fig. 7 Blocking Probability vs. Number of Active Sessions

Fig. 7 shows the blocking probability as a function of the number of active sessions. As expected, the figure shows that the blocking probability increases less steeply as the ratio between thinking time/service (holding) time of the multimedia segment (SEG_mm) increases. It is very important to provide flexibility in the services to be provided by a multimedia distribution system. One of the main performance measure of interest to the users is grade of service that the system may provide, i.e. loss probability. Real-time applications based on audio-visual services may tolerate some losses. The grade of service required will be determined by the application, as well as the coding techniques used [13][14].

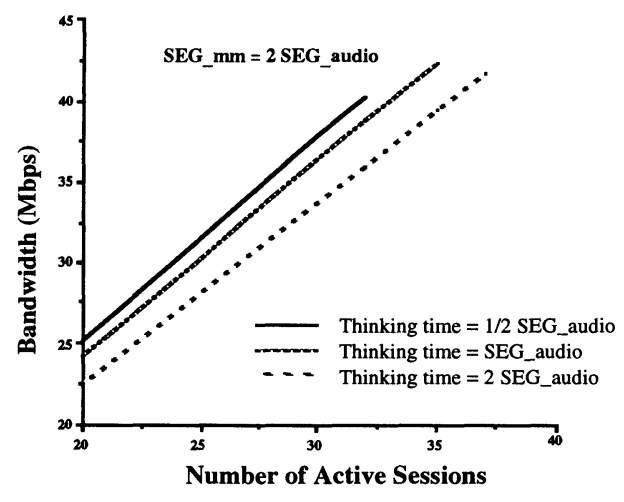

Fig. 8 Bandwidth vs. Number of Active Sessions

In the second part of our analysis, we have been interested in evaluating the impact of introducing a higher degree of visual services. In this second case, the composition of the document is assumed to consist of multimedia segment with a holding time twice the holding time of an audio segment. Fig. 8 shows the bandwidth required as a function of concurrent active sessions. As expected, a higher degree of visual composition of the multimedia 
document severely affects the performance of the system. This results from the fact that, in general, visual services have a more stringent communications needs than audio communications. As for the previous case, Fig. 8 shows the bandwidth required for three different thinking times. The values of the three thinking times considered range from half to twice of the shortest segment namely the segment of type audio. In this case, an improvement of $100 \%$ in the number of concurrent sessions is obtained by using the proposed bandwidth allocation scheme.

Finally, Fig. 9 shows the blocking probability as a function of the active sessions. As in the previous case, this blocking probability gives a measure of the expected probability of being blocked once a user is engaged in the retrieval of a multimedia document. In the case of a realtime application, the fact of offering a degraded service (blocking probability) may be considered as an option for providing service to a larger number of concurrent users.

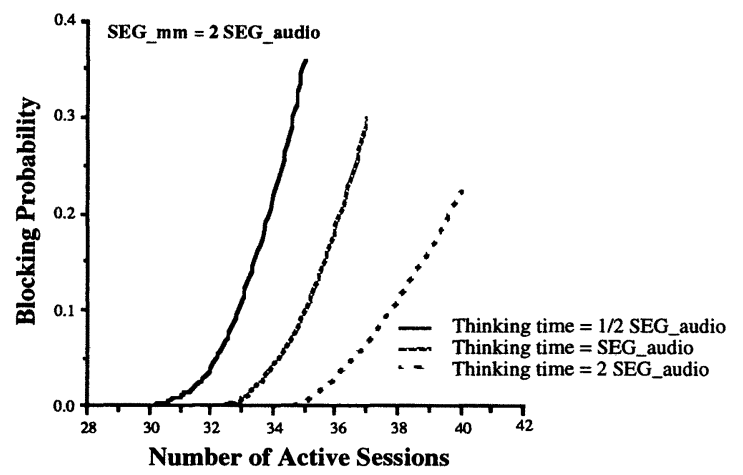

Fig. 9 Blocking Probability vs. Number of Active Sessions

\section{CONCLUSIONS AND FURTHER WORK}

In this paper, we have presented a novel protocol architecture suitable for multimedia document distribution systems. The design of this protocol architecture has been based on the CCITT Standard for Office Document Architecture It has also been assumed the use of the IEEE 802.6 DQDB MAN as the transmission support. The introduction of new protocol elements has been necessary so as to cope with the stringent requirements of a real-time multimedia delivery system, namely : short delays and intermedia synchronization. A performance analysis of the bandwidth allocation scheme has been carried out. Our results show that the blocking probability can be limited for a larger number of sessions as compared to the number of sessions supported by using an static allocation scheme.

The proposed bandwidth allocation scheme can also be considered to be used with other local or metropolitan computer networks providing integrated services, such as FDDI network environments [15][16]. By making use of the multimedia document description (scenario), the scheme can drive the medium access control procedures to allocate the bandwidth according to the requirements of the different media. However, in order to extend this scheme to high speed long haul networks, such as ATM [17], this mechanism can be integrated as part of the procedures used for the establishment of the connection. This last issue requires further study. 


\section{REFERENCES}

[1] L. Orozco-Barbosa and N. D. Georganas, "Multimedia Services and Applications", European Trans on Telecommun. and Related Technologies, vol. II, pp. 5-19, No. JAN/FEB 1/1991.

[2] R. Kositpaiboon, P. Tsingotjidis, L. Orozco-Barbosa. and N.D. Georganas, "Packetized Radiographic Image Transfers over Local Area Networks for Diagnosis and Conferencing", IEEE Journal on Select. Areas on Commun, vol. 7, no. 5, pp. 842856, June 1989.

[3] ISO, Information Processing - Text and Office Systems. Office Document Architecture (ODA) and interchange Format (ODIF), Part one to eight, International Standard 8613, March 1988.

[4] ISO/IEC - Committee Draft International Standard 10744 - Information Technology "Hypermedia/Time-based Structuring Language (HyTime)", April 1991

[5] ISO MHEG Working document "S" - ISO/IEC JTC1/SC29/WG12, Jan. 1992.

[6] N. Borenstein and N. Freed, MIME (Multipurpose Internet Mail Extensions) Mechanisms for Specifying and Describing the Format of Internet Message Bodies, RFC 1341.

[7] ISO-10021(1) and CCITT-X.400. "Message Handling Systems - Information Processing Systems - Text Communication - MOTIS", March 1988.

[8] N. Borenstein and C. Thyberg, "Power, Ease of Use and Cooperative Work in a Practical Multimedia Message System", International Journal of Man-Machine Studies, April 1991.

[9] A. Karmouch, L. Orozco-Barbosa, N.D. Georganas and M. Goldberg, "A Multimedia Medical Communications System,", IEEE Journal on Select. Areas in Commun, vol. 8, no. 3, April 1990, pp. 325-339

[10] IEEE 802.6-1990, IEEE Standards for Local and Metropolitan Area Networks : "Distributed Queue Dual Bus (DQDB) - Subnetwork of a Metropolitan Area Network (MAN)", 1991.

[11] S.S. Lavenberg (Ed.), Computer Performance Modeling Handbook, Academic Press, Inc, San Diego, Cal, 1983.

[12] R. K. Jurgen, "Digital Video", IEEE Spectrum, vol. 29, no. 3, March 1992, pp. 2430.

[13] J.M. Cioffi, J.W.M. Bergmans, H.K. Thapar, and J.K. Wolf (Eds), IEEE Journal on Select. Areas on Commun, Special Issue on Signal Processing and Coding for Recording Channels, vol. 10, no. 1, January 1992.

[14] N. A. Davies and J.R. Nicol, "Technological Perspective on Multimedia Computing", Computer Comms, vol. 14, No. 5, June 1991, pp. 260-272.

[15] ISO 9314-1, Information Processing systems - Fibre Distributed Data Interface (FDDI), Part 1: Token Ring Layer Protocol (PHY), April 1989.

[16] ISO 9314-2, Information Processing systems - Fibre Distributed Data Interface (FDDI), Part 2: Token Ring Media Access Control (MAC), May 1989.

[17] T1S1.5/90-001 R2, Broadband Aspects of ISDN, Baseline Document, June 1990. 